\title{
MATRIX COMPLETIONS, NORMS, AND HADAMARD PRODUCTS
}

\author{
ROY MATHIAS
}

(Communicated by Louis J. Ratliff, Jr.)

\begin{abstract}
Let $M_{m, n}$ (respectively, $H_{n}$ ) denote the space of $m \times n$ complex matrices (respectively, $n \times n$ Hermitian matrices). Let $S \subset H_{n}$ be a closed convex set. We obtain necessary and sufficient conditions for $X_{0} \in S$ to attain the maximum in the following concave maximization problem:
\end{abstract}

$$
\max \left\{\lambda_{\min }(A+X): X \in S\right\}
$$

where $A \in H_{n}$ is a fixed matrix. Let $\circ$ denote the Hadamard (entrywise) product, i.e., given matrices $A=\left[a_{i j}\right], B=\left[b_{i j}\right] \in M_{m, n}$ we define $A \circ B=$ $\left[a_{i j} b_{i j}\right] \in M_{m, n}$.

Using the necessary and sufficient conditions mentioned above we give elementary and unified proofs of the following results.

(a) For any $A \in M_{n, n}$

$$
\omega(A) \equiv \max \left\{\left|x^{*} A x\right|: x \in C^{n}, x^{*} x=1\right\} \leq 1
$$

if and only if there is a matrix $Z \in H_{n}$ such that

$$
\left(\begin{array}{cc}
I+Z & A \\
A^{*} & I-Z
\end{array}\right) \geq 0 .
$$

(b) For any $A \in M_{m, n}$

$$
\max \left\{\|A \circ B\|_{\infty}:\|B\|_{\infty} \leq 1\right\} \leq 1
$$

if and only if there are matrices $P \in H_{m}, Q \in H_{n}$ such that

$$
\left(\begin{array}{cc}
P & A \\
A^{*} & Q
\end{array}\right) \geq 0, \quad P \circ I \leq I, \quad Q \circ I \leq I .
$$

(c) For any $A \in M_{n, n}$

$$
\max \{\omega(A \circ B): \omega(B) \leq 1\} \leq 1
$$

if and only if there is a matrix $P \in H_{n}$ such that

$$
\left(\begin{array}{cc}
P & A \\
A^{*} & P
\end{array}\right) \geq 0, \quad P \circ I \leq I .
$$

We also consider other norms that can be represented in this way and show that if a norm can be represented in this way then so can its dual.

\section{INTRODUCTION AND PRELIMINARIES}

Let $M_{m, n}$ denote the space on $m \times n$ complex matrices and let $M_{n}=$ $M_{n, n}$. Let $H_{n}$ denote the space of complex Hermitian matrices. We use $A \geq$

Received by the editors February 26, 1991 and, in revised form, July 16, 1991.

1991 Mathematics Subject Classification. Primary 15A60, 15A45, 15 A48.

Key words and phrases. Positive semidefinite matrix completion, Hadamard product, Schur product, numerical radius, convex optimization. 
$B$ to mean that $A-B$ is positive semidefinite. Given $A \in M_{m, n}$ define $|A| \equiv\left(A^{*} A\right)^{1 / 2}$. We use $A^{\dagger}$ to denote the Moore-Penrose inverse of $A$. Given $A, B \in M_{m, n}$, the Hadamard product (or Schur product) of $A$ and $B$ is $A$ 。 $B=\left[a_{i j} b_{i j}\right] \in M_{m, n}$. We use $e_{n} \in R^{n}$ to denote the vector of 1's (when the dimension is clear from the context, we use $e$ ). We will consider the following norms:

$$
\begin{aligned}
\|A\|_{\infty} & \equiv \max \left\{x^{*} A y: x \in C^{n}, y \in C^{m}, x^{*} x \leq 1, y^{*} y \leq 1\right\}, \\
\|A\|_{H, \infty} & \equiv \max \left\{\|A \circ B\|_{\infty}: B \in M_{m, n},\|B\| \leq 1\right\}, \\
\omega(A) & \equiv \max \left\{\left|x^{*} A x\right|: x \in C^{n}, x^{*} x \leq 1\right\} \\
\|A\|_{H, \omega} & \equiv \max \left\{\omega(A \circ B): B \in M_{n}, \omega(B) \leq 1\right\} .
\end{aligned}
$$

The first two norms are defined on $M_{m, n}$, the second two are defined on $M_{n}$.

It is a well-known and useful fact (that follows easily from the above definition) that $\|A\|_{\infty} \leq 1$ if and only if

$$
\left(\begin{array}{cc}
I & A \\
A^{*} & I
\end{array}\right) \geq 0
$$

and that $\|A\|_{\infty}=1$ if and only if the given block matrix is positive semidefinite and singular. In this paper we will generalize this result to the norms $\omega(\cdot)$ in $\S 2$, and $\|\cdot\|_{H, \infty}$ and $\|\cdot\|_{H, \omega}$ in $\S 3$. The results in $\S \S 2$ and 3 are not entirely new, however, they are more detailed than existing results and the proofs are constructive and are simpler than the original proofs. These extra details allow us to prove some new result as corollaries. In $\S 4$ we consider generalizations of these results and the corresponding results for dual norms.

Our approach to these characterization problems is to consider the optimality conditions for an appropriate concave maximization problem. These conditions are given in Lemma 1.4. Lemma 1.1 allows us to interpret these conditions in terms of Hadamard products.

There are other results in matrix theory that have been proved by considering the optimality conditions for an appropriate optimization problem. For example, it was shown in [10, p. 230 and Theorem 3.5] that any positive definite matrix $A$ may be written as $A=C \Lambda C$ where $C$ is a correlation matrix and $\Lambda$ is diagonal. It was shown in [8, Theorem 2.6] that if $A \in M_{m, n}$ is entrywise nonnegative then

$$
\|A\|_{\infty}=\min \left\{\left\|\left(B B^{*}\right) \circ I\right\|_{\infty}\left\|\left(C^{*} C\right) \circ I\right\|_{\infty}: B \circ C=A\right\} .
$$

Lemma 1.1. Let $A, B \in H_{n}$ be positive semidefinite. The following are equivalent:

(a) $A B=0$.

(b) $\operatorname{tr} A B=0$.

(c) $e^{*}\left(A \circ B^{\mathrm{T}}\right) e=0$.

Proof. It is immediate that (a) implies (b). To see that (b) implies (a) note that both statements are invariant under a unitary similarity, so we may assume that $A$ is diagonal. The result is now clear, because the main diagonal entries of $B$ are nonnegative and the $i, i$ entry is zero if and only if the $i$ th row and column of $B$ is also 0 . The equivalence of $(b)$ and (c) follows from the fact

$$
\operatorname{tr} A B=\sum_{i, j} a_{i j} \bar{b}_{i j}=\operatorname{tr} e^{*}\left(A \circ B^{\mathrm{T}}\right) e .
$$


The Schur Product Theorem states that if $A, B \in H_{n}$ is positive semidefinite then so is $A \circ B$. The next lemma is a strengthening of the Schur Product Theorem.

Lemma 1.2. Let $A, B \in H_{n}$ be positive semidefinite. Then $A \circ B$ is singular if and only if there is a nonzero diagonal matrix $D$ such that $A D B^{\mathrm{T}} D^{*}=0$.

Proof. By the Schur Product Theorem we know that $A \circ B$ is positive semidefinite. So if $A \circ B$ is singular then there is a nonzero vector $x$ such that $x^{*}(A \circ B) x=0$. Let $D=\operatorname{diag}(x)$. Then

$$
\begin{aligned}
0 & =x^{*}(A \circ B) x=\operatorname{tr}(A \circ B) x x^{*} \\
& =\operatorname{tr}\left(\left(x x^{*}\right)^{\mathrm{T}} \circ A\right) B^{\mathrm{T}}=\operatorname{tr}\left(D^{*} A D\right) B^{\mathrm{T}}=\operatorname{tr} A\left(D^{*} B D\right)
\end{aligned}
$$

and hence by Lemma 1.1 we have $A\left(D^{*} B D\right)=0$. For the third equality we have used the fact (which can be verified computationally) that for any matrices $X, Y, Z \in M_{n}$

$$
\operatorname{tr}(X \circ Y) Z=\operatorname{tr}\left(X \circ Z^{\mathrm{T}}\right) Y^{\mathrm{T}} .
$$

To prove the converse reverse this argument.

Given a convex cone $K \subset H_{n}$ define its polar cone $K^{*}$ by

$$
K^{*}=\left\{A \in H_{n}: \operatorname{tr} A B^{*} \leq 0 \text { for all } B \in K\right\} .
$$

We state the following theorem of the alternative as we will use it in an essential way twice. It is an analog in $H_{n}$ of a standard theorem of the alternative for convex sets in $R^{n}$ and can be derived in the same way (see, e.g., [7, $§ 3.2$, Theorem 6]).

Theorem 1.3. Let $K \subset H_{n}$ be a convex cone and $W \subset H_{n}$ be a compact set. Then exactly one of the following holds:

(a) There is a matrix $A \in K$ such that $\operatorname{tr} A B>0$ for all $B \in W$.

(b) There are $\lambda_{i}>0$ and $B_{i} \in W$ such that $\sum_{i=1}^{k} \lambda_{i} B_{i} \in K^{*}$.

Optimality conditions similar to those in the next lemma are given in [12,3].

Lemma 1.4. Let $S$ be a closed convex subset of $H_{n}$, and let $A \in H_{n}$ and $X_{0} \in S$ be given. Let $K$ be the cone of feasible directions at $X_{0}$. Then the following are equivalent:

(a) $\lambda_{\min }\left(A+X_{0}\right) \geq \lambda_{\min }(A+X)$ for all $X \in S$.

(b) There is a nonzero positive semidefinite matrix $M \in K^{*}$ such that

$$
\left(A+X_{0}\right) M=\lambda_{\min }\left(A+X_{0}\right) M .
$$

If $S$ is a subspace of $H_{n}$ then $K^{*}=S^{\perp}$ independent of $X_{0}$.

Proof. Without loss of generality we may assume that $\lambda_{\min }\left(A+X_{0}\right)=0$. Let

$$
N=\left\{y \in C^{n}:\left(A+X_{0}\right) y=0 \text { and } y^{*} y=1\right\}
$$

and

$$
C=\left\{y y^{*}: y \in N\right\} .
$$

Then $C$ is a compact set. Also,

$$
\lambda_{\min }\left(A+X_{0}\right) \geq \lambda_{\min }(A+X) \text { for all } X \in S
$$


if and only if there is no $X \in S$ such that

$$
\operatorname{tr}\left(X-X_{0}\right) y y^{*}=y^{*}\left(X-X_{0}\right) y>0 \text { for all } y \in N,
$$

or equivalently

$$
\nexists D \in K \text { such that } \operatorname{tr} D Y>0 \text { for all } Y \in C \text {. }
$$

However, because $K$ is a convex cone and $C$ is a compact set, the statement (1.1) is equivalent, by Theorem 1.3 , to

$$
\exists y_{i} \in N, \lambda_{i}>0 \text { such that } 0 \neq \sum_{i=1}^{k} \lambda_{k} y_{i} y_{i}^{*} \in K^{*} .
$$

This completes the proof as $M$ is a nonzero positive semidefinite matrix such that $\left(A+X_{0}\right) M=0$ if and only if $M=\sum_{i=1}^{k} \lambda_{i} y_{i} u y_{i}^{*}$ for some $k>0, y_{i} \in N$, and $\lambda_{i}>0$.

\section{THE NUMERICAL RADIUS}

In this section we give an elementary proof of a useful characterization of matrices that have numerical radius at most 1. Part (a) of Theorem 2.1 is due to Ando [1].

Theorem 2.1. Let $A \in M_{n}$.

(a) $\omega(A) \leq 1$ if and only if there is a matrix $Z \in H_{n}$ such that

$$
\left(\begin{array}{cc}
I+Z & A \\
A^{*} & I-Z
\end{array}\right) \geq 0 .
$$

(b) Suppose that $Z \in H_{n}$ is such that

$$
\left(\begin{array}{cc}
I+Z & A \\
A^{*} & I-Z
\end{array}\right) \geq 0 .
$$

Then $\omega(A)=1$ if and only if there is a vector $x \in C^{n}$ and a scalar $\alpha \in C$ with $x^{*} x=|\alpha|=1$ such that

$$
\left(\begin{array}{cc}
I+Z & A \\
A^{*} & I-Z
\end{array}\right)\left(\begin{array}{c}
x \\
\alpha x
\end{array}\right)=\left(\begin{array}{l}
0 \\
0
\end{array}\right)
$$

In this case $\left|x^{*} A x\right|=1$.

(c) $\omega(A)=1$ if and only if there is a $2 n \times 2 n$ matrix

$$
\left(\begin{array}{ll}
Y_{11} & Y_{12} \\
Y_{12}^{*} & Y_{11}
\end{array}\right) \geq 0, \quad Y_{i j} \in M_{n},
$$

and a matrix $Z \in H_{n}$ such that

$$
B \equiv\left(\begin{array}{cc}
I+Z & A \\
A^{*} & I-Z
\end{array}\right) \geq 0
$$

and

$$
\left(\begin{array}{cc}
I+Z & A \\
A^{*} & I-Z
\end{array}\right)\left(\begin{array}{cc}
Y_{11} & Y_{12} \\
Y_{12}^{*} & Y_{11}
\end{array}\right)=0
$$


Let $Y$ be any such matrix, and let rank $Y=k$. Then there is a matrix $R \in M_{n, k}$ with nonzero columns and a diagonal unitary matrix $D \in M_{k}$ such that

$$
\left(\begin{array}{c}
R \\
R D
\end{array}\right)\left(\begin{array}{c}
R \\
R D
\end{array}\right)^{*}=\left(\begin{array}{ll}
Y_{11} & Y_{12} \\
Y_{12}^{*} & Y_{11}
\end{array}\right)
$$

and for any column $r_{i}$ of $R$ we have $r_{i} \neq 0$ and $\left|r_{i}^{*} A r_{i}\right|=r_{i}^{*} r_{i}$.

The main content of this theorem can be restated as

$$
-\omega(A)=\max _{Z \in H_{n}} \lambda_{\min }\left(\begin{array}{cc}
Z & A \\
A^{*} & -Z
\end{array}\right) .
$$

In [1] Ando proves more than part (a) of the theorem. He shows that if $\omega(A) \leq$ 1 then the set

$$
\left\{Z \in H_{n}:\left(\begin{array}{cc}
I+Z & A \\
A^{*} & I-Z
\end{array}\right) \geq 0\right\}
$$

has a minimal and maximal element (with respect to the partial order $\geq$ on $H_{n}$ ). It appears that our approach does not yield this additional information.

Proof. We will first show that if there is a matrix $Z$ for which (2.1) holds then $\omega(A) \leq 1$. Let $Z$ be such a matrix and take any $x \in C^{n}$ and $\alpha \in C$ with $x^{*} x=|\alpha|=1$. Then

$$
0 \leq\left(\begin{array}{c}
x \\
\alpha x
\end{array}\right)^{*}\left(\begin{array}{cc}
I+Z & A \\
A^{*} & I-Z
\end{array}\right)\left(\begin{array}{c}
x \\
\alpha x
\end{array}\right)=2+2 \operatorname{Re}\left(\alpha x^{*} A x\right) .
$$

Hence $\omega(A) \leq 1$. This calculation also establishes (b).

Now we will prove (c) and the other direction of (a) by considering

$$
c(A) \equiv \max _{Z \in H_{n}} \lambda_{\min }\left(\left(\begin{array}{cc}
I+Z & A \\
A^{*} & I-Z
\end{array}\right)\right) .
$$

Let us first consider the case $c(A)=0$. The maximization is over the subspace $S=\left\{Z \oplus(-Z): Z \in H_{n}\right\}$. It is easy to show that

$$
S^{\perp}=\left\{\left(\begin{array}{ll}
Y_{11} & Y_{12} \\
Y_{12}^{*} & Y_{11}
\end{array}\right) \in H_{2 n}: Y_{i i} \in M_{n}\right\} .
$$

So by Lemma 1.4 this maximum is 0 and is attained at the matrix $Z$ if and only if there is a matrix of the form (2.2) such that (2.3) holds. Let $Y$ be such a matrix and let rank $Y=k$. Then, because $Y \geq 0$,

$$
\left(\begin{array}{ll}
Y_{11} & Y_{12} \\
Y_{12}^{*} & Y_{11}
\end{array}\right)=\left(\begin{array}{l}
R_{1} \\
R_{2}
\end{array}\right)\left(\begin{array}{l}
R_{1} \\
R_{2}
\end{array}\right)^{*}
$$

for some matrices $R_{i} \in M_{n, k}$. Because $R_{1} R_{1}^{*}=R_{2} R_{2}^{*}$, there is a unitary matrix $U \in M_{k}$ such that $R_{1} U=R_{2}$. Let $U=V D V^{*}$ where $V, D$ are unitary and $D$ is diagonal. Let $R=R_{1} V$. Then

$$
0=\left(\begin{array}{cc}
I+Z & A \\
A^{*} & I-Z
\end{array}\right)\left(\begin{array}{l}
R_{1} \\
R_{2}
\end{array}\right)
$$

and hence

$$
0=\left(\begin{array}{cc}
I+Z & A \\
A^{*} & I-Z
\end{array}\right)\left(\begin{array}{l}
R_{1} V \\
R_{2} V
\end{array}\right)=\left(\begin{array}{cc}
I+Z & A \\
A^{*} & I-Z
\end{array}\right)\left(\begin{array}{c}
R \\
R D
\end{array}\right)
$$


Because rank $Y=k$, every column of $R$ must be nonzero. By part (b) it follows that $\left|r_{i}^{*} A r_{i}\right|=r_{i}^{*} r_{i}$ and that $\omega(A)=1$. We have now established (c) and shown that if $c(A)=0$ then $\omega(A)=1$.

Now let us prove the 'only if' part of (a). Suppose that there is no matrix $Z \in H_{n}$ for which (2.1) holds. Then $c(A)<0$. Straightforward manipulations show that if $c(A)<0$ then $c\left([1-c(A)]^{-1} A\right)=0$. So by the preceding analysis, $\omega\left([1-c(A)]^{-1} A\right)=1$ or, equivalently, $\omega(A)=1-c(A)$. (The identity (2.4) follows from this.) So since $c(A)<0$, we have $\omega(A)=1-c(A)>1$. This proves the 'only if' part of (a).

The power inequality for the numerical radius states that if $\omega(A) \leq 1$ then $\omega\left(A^{k}\right) \leq 1$ for any positive integer $k$. (It is not true, in general, that $\omega(A B) \leq$ $\omega(A) \omega(B)$.) This inequality follows easily from Theorem 2.1. For if $\omega(A) \leq 1$ then there is a Hermitian contraction $Z$ such that

$$
\left(\begin{array}{cc}
I+Z & A \\
A^{*} & I-Z
\end{array}\right) \geq 0
$$

and hence there is a contraction $C$ such that

$$
A=(I+Z)^{1 / 2} C(I-Z)^{1 / 2} \text {. }
$$

So for any positive integer $k$

$$
\begin{aligned}
A^{k} & =(I+Z)^{1 / 2} C\left[(I+Z)^{1 / 2}(I-Z)^{1 / 2} C\right]^{k-1}(I-Z)^{1 / 2} \\
& =(I+Z)^{1 / 2} C\left[\left(I-Z^{2}\right)^{1 / 2} C\right]^{k-1}(I-Z)^{1 / 2} .
\end{aligned}
$$

Because $C$ and $Z$ are contractions and $Z$ is Hermitian, $C\left[\left(I-Z^{2}\right)^{1 / 2} C\right]^{k-1}$ is also a contraction, and hence

$$
\left(\begin{array}{cc}
I+Z & A^{k} \\
\left(A^{k}\right)^{*} & I-Z
\end{array}\right) \geq 0
$$

from which it follows that $\omega\left(A^{k}\right) \leq 1$.

\section{HADAMARD PRODUCTS}

In this section we consider the norms $\|\cdot\|_{H, \infty}$ and $\|\cdot\|_{H, \omega}$. The results in Theorem 3.1 can be derived from this in Theorem 3.2 by using the following identity [2, Lemma 8]:

$$
\|A\|_{H, \infty}=\left\|\left(\begin{array}{cc}
0 & A \\
0 & 0
\end{array}\right)\right\|_{H, \omega}
$$

(the 0 blocks are $n \times n$ ). However, it is simpler to prove Theorem 3.1 directly. Theorem 3.1(a) is due to Haagerup (unpublished); it was proved, in a more general form (involving partial matrices), in [13, Theorem 3.2]; it was also derived by Ando and Okubo, using the identity above, from the main result in [2], which is Theorem 3.2(a). All these proofs are based on Arvenson's Extension Theorem for positive maps on $C^{*}$ algebras.

Theorem 3.1. Let $A \in M_{m, n}$.

(a) $\|A\|_{H, \infty} \leq 1$ if and only if there are matrices $P \in H_{m}$ and $Q \in H_{n}$ such that

$$
\left(\begin{array}{cc}
P & A \\
A^{*} & Q
\end{array}\right) \geq 0, \quad P \circ I \leq I, \quad Q \circ I \leq I .
$$


(b) Suppose that $P \in H_{m}, Q \in H_{n}$ are such that (3.1) holds. Then $\|A\|_{H, \infty}=$ 1 if and only if there are matrices $D_{1} \in M_{m}, D_{2} \in M_{n}$, and $Y \in M_{m, n}$ with $D_{1}$ and $D_{2}$ diagonal and such that

$$
\begin{gathered}
\left(\begin{array}{cc}
D_{1} & Y \\
Y^{*} & D_{2}
\end{array}\right) \geq 0, \quad\left(\begin{array}{cc}
P & A \\
A^{*} & Q
\end{array}\right)\left(\begin{array}{cc}
D_{1} & Y \\
Y^{*} & D_{2}
\end{array}\right)=0, \\
D_{1}(P \circ I-I)=0, \quad D_{2}(Q \circ I-I)=0 .
\end{gathered}
$$

For any such $D_{1}, D_{2}$, and $Y$

$$
\sigma_{1}\left(D_{1}^{1 / 2 \dagger} \bar{Y} D_{2}^{1 / 2 \dagger}\right)=1 \quad \text { and } \quad \sigma_{1}\left(A \circ\left[D_{1}^{1 / 2 \dagger} \bar{Y} D_{2}^{1 / 2 \dagger}\right]\right)=1 .
$$

In particular,

$$
y^{*}\left(A \circ\left[D_{1}^{1 / 2 \dagger} \bar{Y} D_{2}^{1 / 2 \dagger}\right]\right) z=\sqrt{y^{*} y} \sqrt{z^{*} z}
$$

where $y=D_{1}^{1 / 2} e_{m}$ and $z=D_{2}^{1 / 2} e_{n}$.

Note that condition (3.3) is equivalent to $D_{1} \circ P=D_{1}$ and $D_{2} \circ Q=D_{2}$.

Proof. Let $A \in M_{m, n}$ be given. Suppose that there are matrices $P \in H_{m}$ and $Q \in H_{n}$ such that (3.1) holds. Let $X \in M_{n}$ be such that $\|X\|_{\infty} \leq 1$. Then

$$
\left(\begin{array}{cc}
I & X \\
X^{*} & I
\end{array}\right) \geq 0
$$

Now by the Schur Product Theorem and the fact that $P \circ I \leq I$ and $Q \circ I \leq I$, we have

$$
0 \leq\left(\begin{array}{cc}
I \circ P & X \circ A \\
X^{*} \circ A^{*} & I \circ P
\end{array}\right) \leq\left(\begin{array}{cc}
I & A \circ X \\
(A \circ X)^{*} & I
\end{array}\right) .
$$

Hence $\|A \circ X\|_{\infty} \leq 1$. So $\|A\|_{H, \infty} \leq 1$.

To prove the other direction and the second statement consider

$$
c(A) \equiv \max \left\{\lambda_{\min }\left(\begin{array}{cc}
P & A \\
A^{*} & Q
\end{array}\right): P \in H_{n}, Q \in H_{m}, P \circ I \leq I, \text { and } Q \circ I \leq I\right\} \text {. }
$$

The maximum is attained by a compactness argument. Let us first consider the case $c(A)=0$. By Lemma 1.4 this maximum is 0 and is attained at the matrices $P, Q$ if and only if there are matrices $D_{1}, D_{2}, Y$ such that (3.2) and (3.3) hold. The first inequality in (3.2) yields

$$
\left(\begin{array}{cc}
I & D_{1}^{1 / 2 \dagger} \bar{Y} D_{2}^{1 / 2 \dagger} \\
\left(D_{1}^{1 / 2 \dagger} \frac{I}{Y} D_{2}^{1 / 2 \dagger}\right)^{*} & I
\end{array}\right) \geq 0
$$

so $\left\|D_{1}^{1 / 2 \dagger} \bar{Y} D_{2}^{1 / 2 \dagger}\right\|_{\infty} \leq 1$. By the second condition in (3.2) and Lemma 1.1, we have

$$
e_{2 n}^{*}\left(\begin{array}{cc}
D_{1} \circ P & A \circ \bar{Y} \\
(A \circ \bar{Y})^{*} & D_{2} \circ Q
\end{array}\right) e_{2 n}=0 .
$$

The complementarity conditions (3.3) imply $D_{1} \circ P=D_{1}$ and $D_{2} \circ Q=D_{2}$. So

$$
e_{2 n}^{*}\left(\begin{array}{cc}
D_{1} & A \circ \bar{Y} \\
(A \circ \bar{Y})^{*} & D_{2}
\end{array}\right) e_{2 n}=0 .
$$

Since the matrix in this expression is positive semidefinite, $\left\|A \circ\left[D_{1}^{1 / 2 \dagger} \bar{Y} D_{2}^{1 / 2 \dagger}\right]\right\|_{\infty}$ $=1$ and, consequently, $\|A\|_{H, \infty}=1$. 
Now suppose that there are no matrices $P, Q$ such that (3.1) holds. Then $c(A)<0$. The rest of the proof, i.e., showing the 'only if' part of $(\mathrm{a})$, is the same as the end of the proof of the previous theorem.

Theorem 3.2. Let $A \in M_{n}$.

(a) $\|A\|_{H, \omega} \leq 1$ if and only if there is a matrix $P \in M_{n}$ such that

$$
\left(\begin{array}{cc}
P & A \\
A^{*} & P
\end{array}\right) \geq 0 \text { and } P \circ I \leq I .
$$

(b) Suppose that $P \in H_{n}$ is such that (3.4) holds. Then $\|A\|_{H, \omega}=1$ if and only if there are matrices $D_{1}, D_{2}, H \in M_{n}$ and $Y \in M_{n}$ with $D_{1}$ and $D_{2}$ diagonal and $H$ Hermitian with main diagonal zero such that

$$
\begin{gathered}
\left(\begin{array}{cc}
D_{1}+H & Y \\
Y^{*} & D_{2}-H
\end{array}\right) \geq 0, \quad\left(\begin{array}{cc}
P & A \\
A^{*} & P
\end{array}\right)\left(\begin{array}{cc}
D_{1}+H & Y \\
Y^{*} & D_{2}-H
\end{array}\right)=0, \\
\Delta(P \circ I-I)=0
\end{gathered}
$$

(where $\Delta=\left(D_{1}+D_{2}\right) / 2$ ). For any such $D_{1}, D_{2}, H$, and $Y$

$$
\omega\left(\Delta^{\dagger 1 / 2} \bar{Y} \Delta^{\dagger 1 / 2}\right)=1 \text { and } \omega\left(A \circ\left(\Delta^{\dagger 1 / 2} \bar{Y} \Delta^{\dagger 1 / 2}\right)\right)=1 .
$$

In particular,

$$
y^{*}\left(A \circ\left[\Delta^{\dagger 1 / 2} \bar{Y} \Delta^{\dagger 1 / 2}\right]\right) y=y^{*} y
$$

where $y=\Delta^{1 / 2} e_{n}$.

Proof. Let $A \in M_{n}$ be given. Suppose that there is a matrix $P \in H_{n}$ such that (3.4) holds. Let $X \in M_{n}$ be such that $\omega(X) \leq 1$. Then, by Theorem 2.1, there is $Z \in H_{n}$ such that

$$
\left(\begin{array}{cc}
I+Z & X \\
X^{*} & I-Z
\end{array}\right) \geq 0 .
$$

Now by the Schur Product Theorem and the fact that $P \circ I \leq I$, we have

$$
0 \leq\left(\begin{array}{cc}
(I+Z) \circ P & X \circ A \\
X^{*} \circ A^{*} & (I-Z) \circ P
\end{array}\right) \leq\left(\begin{array}{cc}
I+P \circ Z & A \circ X \\
(A \circ X)^{*} & I-P \circ Z
\end{array}\right) .
$$

Hence, by Theorem 2.1 again, $\omega(A \circ X) \leq 1$. So $\|A\|_{H, \omega} \leq 1$.

To prove the other direction and the second statement consider

$$
c(A) \equiv \max \left\{\lambda_{\min }\left(\begin{array}{cc}
P & A \\
A^{*} & P
\end{array}\right): P \in H_{n} \text { and } P \circ I \leq I\right\} .
$$

The maximum is attained by a compactness argument. First consider the case $c(A)=0$. By Lemma 1.4 this maximum is 0 and is attained at the matrix $P$ if and only if there are matrices $D_{1}, D_{2}, H, Y$ such that (3.5) and (3.6) hold. Let $\Delta=\left(D_{1}+D_{2}\right) / 2$ and $D_{3}=\left(D_{1}-D_{2}\right) / 2$. Then

$$
\left(\begin{array}{cc}
I+\Delta^{\dagger 1 / 2}\left(D_{3}+H\right) \Delta^{\dagger 1 / 2} & \Delta^{\dagger 1 / 2} Y \Delta^{\dagger 1 / 2} \\
\left(\Delta^{\dagger 1 / 2} Y \Delta^{\dagger 1 / 2}\right)^{*} & I-\Delta^{\dagger 1 / 2}\left(D_{3}+H\right) \Delta^{\dagger 1 / 2}
\end{array}\right) \geq 0
$$

so, by Theorem 2.1, $\omega\left(\Delta^{\dagger 1 / 2} \bar{Y} \Delta^{\dagger 1 / 2}\right) \leq 1$. Also, by the second condition in (3.5) and Lemma 1.1 , we have

$$
e_{2 n}^{*}\left(\begin{array}{cc}
D_{1}+P \circ \bar{H} & A \circ \bar{Y} \\
(A \circ \bar{Y})^{*} & D_{2}-P \circ \bar{H}
\end{array}\right) e_{2 n}=0
$$


or, equivalently,

$$
\left(\begin{array}{c}
\Delta^{1 / 2} e_{n} \\
\Delta^{1 / 2} e_{n}
\end{array}\right)^{*}\left(\begin{array}{cc}
I+\Delta^{\dagger 1 / 2}\left(P \circ \bar{H}+D_{3}\right) \Delta^{\dagger 1 / 2} & A \circ \Delta^{\dagger 1 / 2} \bar{Y} \Delta^{\dagger 1 / 2} \\
\left(A \circ \Delta^{\dagger 1 / 2} \bar{Y} \Delta^{\dagger 1 / 2}\right)^{*} & I-\Delta^{\dagger 1 / 2}\left(P \circ \bar{H}+D_{3}\right) \Delta^{\dagger 1 / 2}
\end{array}\right)\left(\begin{array}{c}
\Delta^{1 / 2} e_{n} \\
\Delta^{1 / 2} e_{n}
\end{array}\right)=0 .
$$

By Lemma 2.1 above or a straightforward computation, this implies that $\omega\left(A \circ\left[\Delta^{\dagger 1 / 2} \bar{Y} \Delta^{\dagger 1 / 2}\right]\right) \geq 1$ and that $\|A\|_{H, \omega}=1$. The rest of the proof is the same as that of Theorem 3.1.

The rest of this section is devoted to corollaries of Theorems 3.1 and 3.2.

The following result is new. It is natural and follows easily from the constructive proofs given in the previous section. The identity (3.7) verifies Conjecture 6.10 in [9].

Corollary 3.3. Let $A \in M_{m, n}(R)$ and $B \in M_{n}(R)$. Then

$$
\|A\|_{H, \infty}=\max \left\{\|A \circ X\|_{\infty}:\|X\|_{\infty} \leq 1 \text { and } X \in M_{m, n}(R)\right\}
$$

and

$$
\|A\|_{H, \omega}=\max \left\{\omega(A \circ X): \omega(X) \leq 1 \text { and } X \in M_{n}(R)\right\} .
$$

Let $A \in H_{n}$. Then

$$
\|A\|_{H, \infty}=\max \left\{\|A \circ X\|_{\infty}:\|X\|_{\infty} \leq 1 \text { and } X \in H_{n}\right\}
$$

and

$$
\|A\|_{H, \omega}=\max \left\{\omega(A \circ X): \omega(X) \leq 1 \text { and } X \in H_{n}\right\} .
$$

Proof. The proofs of the results for $A$ real are very similar to the case where $A$ is Hermitian and are omitted. In the Hermitian case we will prove the result for the norm $\|\cdot\|_{H, \omega}$. The result for $\|\cdot\|_{H, \infty}$ follows from this because $\|A\|_{H, \infty} \leq\|A\|_{H, \omega}$ for any $A \in M_{n}$. (In fact, it is easy to show from the representations in Theorems 3.1 and 3.2 that $\|A\|_{H, \infty}=\|A\|_{H, \omega}$ if $A=A^{*}$.

See [2, Corollary 3] for the details.)

Let $A \in H_{n}$ be given. We may assume that $\|A\|_{H, \omega}=1$. Then by Theorem 3.2 there are matrices $D_{1}, D_{2}, P, H, Y$ such that

$$
\left(\begin{array}{cc}
D_{1}+H & Y \\
Y^{*} & D_{2}-H
\end{array}\right) \geq 0, \quad\left(\begin{array}{cc}
P & A \\
A^{*} & P
\end{array}\right) \geq 0
$$

and

$$
\left(\begin{array}{cc}
P & A \\
A^{*} & P
\end{array}\right)\left(\begin{array}{cc}
D_{1}+H & Y \\
Y^{*} & D_{2}-H
\end{array}\right)=0 .
$$

However, because $A=A^{*}$, applying a block permutation similarity to equation (3.9) gives

$$
\left(\begin{array}{cc}
P & A \\
A^{*} & P
\end{array}\right)\left(\begin{array}{cc}
D_{2}-H & Y^{*} \\
Y & D_{1}+H
\end{array}\right)=0
$$

Adding these two equations gives

$$
\left(\begin{array}{cc}
P & A \\
A^{*} & p
\end{array}\right)\left(\begin{array}{cc}
D_{1}+D_{2} & Y+Y^{*} \\
Y+Y^{*} & D_{1}+D_{2}
\end{array}\right)=0
$$

It is clear that

$$
\left(\begin{array}{cc}
D_{1}+D_{2} & Y+Y^{*} \\
Y+Y^{*} & D_{1}+D_{2}
\end{array}\right)
$$


being the sum of two positive semidefinite matrices is itself positive semidefinite. Thus, by Theorem 2.1,

$$
\omega\left(A \circ\left[\left(D_{1}+D_{2}\right)^{\dagger 1 / 2}\left(Y+Y^{*}\right)^{\mathrm{T}}\left(D_{1}+D_{2}\right)^{\dagger 1 / 2}\right]\right)=1 .
$$

Since the matrix $\left(D_{1}+D_{2}\right)^{\dagger 1 / 2}\left(Y+Y^{*}\right)^{\mathrm{T}}\left(D_{1}+D_{2}\right)^{\dagger 1 / 2}$ is Hermitian, (3.8) follows.

It is clear from Theorem 3.1 that $\|A\|_{H, \infty} \leq\|A\|_{\infty}$ for any matrix $A$. The case of equality was characterized in [11]; we show that the characterization follows easily from Theorem 3.1 .

Corollary 3.4. Let $\|A\|_{\infty}=1$. Then $\|A\|_{H, \infty}=1$ if and only if a submatrix of $A$ is unitary.

Proof. Let $A \in M_{n}$ with $\|A\|_{\infty}=1$ be given. Then

$$
\left(\begin{array}{cc}
I & A \\
A^{*} & I
\end{array}\right) \geq 0
$$

If $\|A\|_{H, \infty}=1$ then by the second statement in Theorem 3.1 there must be matrices $D_{1}, D_{2}, Y$ of the appropriate dimensions such that

$$
\left(\begin{array}{cc}
D_{1} & Y \\
Y^{*} & D_{2}
\end{array}\right) \geq 0 \text { and }\left(\begin{array}{cc}
I & A \\
A^{*} & I
\end{array}\right)\left(\begin{array}{cc}
D_{1} & Y \\
Y^{*} & D_{2}
\end{array}\right)=0
$$

Assume, without loss of generality, that the first $k_{1}$ (respectively, $k_{2}$ ) main diagonal entries of $D_{1}$ (respectively, $D_{2}$ ) are positive and the remaining entries are 0 . Let $\widehat{D}_{1}$ (respectively, $\widehat{D}_{2}, \widehat{Y}, \widehat{A}$ ) be the $k_{1} \times k_{1}$ (respectively, $k_{2} \times$ $k_{2}, k_{1} \times k_{2}, k_{1} \times k_{2}$ ) submatrix in the upper left corner of $D_{1}$ (respectively, $\left.D_{2}, Y, A\right)$. Then

$$
\left(\begin{array}{cc}
I_{k_{1}} & \widehat{A} \\
\widehat{A}^{*} & I_{k_{2}}
\end{array}\right)\left(\begin{array}{ll}
\widehat{D}_{1} & \widehat{Y} \\
\widehat{Y}^{*} & \widehat{D}_{2}
\end{array}\right)=0 .
$$

Consideration of the 1,1 and 2,1 entries in the product gives

$$
\widehat{D}_{1}=-\widehat{A} \widehat{Y}^{*} \text { and } \widehat{Y}^{*}=-\widehat{A}^{*} \widehat{D}_{1} \text {. }
$$

So $\widehat{D}_{1}=\widehat{A} \widehat{A}^{*} \widehat{D}_{1}$, and now by the nonsingularity of $\widehat{D}_{1}$ we have $I_{k_{1}}=\widehat{A} \widehat{A}^{*}$. Similarly, $I_{k_{2}}=\widehat{A}^{*} \widehat{A}$. Thus $\widehat{A}$ must be unitary.

\section{Generalizations and dUAl NORMS}

In this section we generalize the ideas of $\S \S 2$ and 3 and relate the results to those for dual norms. Given a norm $\|\cdot\|$ on $M_{m, n}$, we define its dual norm by $\|A\|^{*}=\max \left\{\left|\operatorname{tr} A B^{*}\right|:\|B\| \leq 1\right\}$.

Theorem 4.1. Let $m, n$ be given positive integers. Let $S$ be a closed convex subset of $H_{m+n}$ such that any $P \in S$ may be written $P_{1} \oplus P_{2}$ where $P_{1} \in H_{m}$ and $P_{2} \in H_{n}$. Define a function $N(\cdot)$ on $M_{m, n}$ by

$$
N(A)=\inf \left\{\alpha \geq 0: \alpha P+\left(\begin{array}{cc}
0 & A \\
A^{*} & 0
\end{array}\right) \geq 0 \text { for some } P \in S\right\} .
$$

Then $N(\cdot)$ is a norm if and only if

(a) there is a positive definite matrix $P \in S$;

(b) $S_{+}=S \cap\{P \geq 0\}$ is compact.

In this case the infimum in (4.1) is attained for any $A \in M_{m, n}$. 
Proof. First we show that if conditions (a) and (b) hold then $N(\cdot)$ is a norm. It is clear that $N(\beta A)=|\beta| N(A)$ and that $N(A+B) \leq N(A)+N(B)$ for any $A, B \in M_{m, n}$ and any $\beta \in C$ and that $N(0)=0$. So in order to show that $N(\cdot)$ is a norm it suffices to show that $N(A) \in(0, \infty)$ for any nonzero $A \in M_{m, n}$.

Let $A \in M_{m, n}$ be a given nonzero matrix. By condition (a) there is an $\varepsilon>0$ $P \in S$ such that $P \geq \varepsilon I$. So

$$
\left(\varepsilon^{-1}\|A\|_{\infty}\right) P+\left(\begin{array}{cc}
0 & A \\
A^{*} & 0
\end{array}\right) \geq\left(\varepsilon^{-1}\|A\|_{\infty}\right) I+\left(\begin{array}{cc}
0 & A \\
A^{*} & 0
\end{array}\right) \geq 0,
$$

and hence $N(A) \leq \varepsilon^{-1}\|A\|_{\infty}<\infty$. By condition (b) there is a positive constant $c$ such that $P \leq c I$ for all $P \in S$. For any $\alpha<C^{-1}\|A\|_{\infty}$ we have

$$
\alpha P+\left(\begin{array}{cc}
0 & A \\
A^{*} & 0
\end{array}\right)<\|A\|_{\infty} I+\left(\begin{array}{cc}
0 & A \\
A^{*} & 0
\end{array}\right) .
$$

The right-hand side is not positive definite and hence the left-hand side cannot be positive semidefinite. Thus $N(A) \geq c^{-1}\|A\|_{\infty}$. We showed that $N(\cdot)$ is a norm. By the second condition the set $\{P \in S: P \geq 0\}$ is a compact set, and hence the infimum is attained for any $A \in M_{m, n}$.

Now we show that if $N(\cdot)$ is a norm then conditions (a) and (b) must be satisfied. First we show that (a) is necessary.

Let $z \in C^{m+n}$ be a given nonzero vector. We will show that there is a matrix $P \in S_{+}$such that $z^{*} P z>0$. Write $z=\left(\begin{array}{l}x \\ y\end{array}\right)$ with $x \in C^{m}$ and $y \in C^{m}$. Without loss of generality we may assume that $y$ is not 0 . Because $N\left(e_{m} y^{*}\right)=c<\infty$ there must be a matrix $P=P_{1} \oplus P_{2} \in S_{+}$such that

$$
\left(\begin{array}{cc}
c P_{1} & e_{m} y^{*} \\
y e_{m}^{*} & c P_{2}
\end{array}\right) \geq 0
$$

This implies that $P_{1}$ is positive semidefinite and that $y$ is in the range of $P_{2}$ and hence $y^{*} P_{2} y>0$. So $z^{*} P z^{*}=x^{*} P_{1} x+y^{*} P_{2} y \geq y^{*} P_{2} y>0$. Thus we showed that for each unit vector $z \in C^{m+n}$ there is a matrix $P \in S$ such that $z^{*} P z>0$; that is,

$$
\nexists \lambda_{i}>0, z_{i} \in C^{m+n} \text { with } z_{i}^{*} z_{i}=1 \text { such that } \sum_{i=1}^{k} \lambda_{i} z_{i} z_{i}^{*} \in S^{*} .
$$

By Theorem 1.3, this is equivalent to

$$
\exists P \in S \text { such that } z^{*} P z^{*}>0 \text { for all } z \in C^{m+n}, z^{*} z=1 .
$$

Thus we showed that if $N(\cdot)$ is a norm then $S$ must contain a positive definite matrix.

We now show that if $N(\cdot)$ is a norm then $S_{+}$must be compact. Since $S_{+}$ is closed, it is sufficient to show that $S_{+}$is bounded. Let $Q \in S$ be positive definite and let $a=\lambda_{\min }(Q)>0$. Then $Q \geq a I$. Let $c>0$ be such that

$$
N(A) \geq c\|A\|_{\infty} \quad \text { for all } A \in M_{m, n} .
$$

The existence of such a positive constant is guaranteed by the equivalence of the norms $N(\cdot)$ and $\|\cdot\|_{\infty}$ on the finite-dimensional vector space $M_{m, n}[4$, 
Corollary 5.4.5]. Let $P \in S_{+}$be given and take any $x \in C^{m}$. Let $y=$ $[1,0, \ldots, 0]^{*} \in C^{n}$. Then $N\left(x y^{*}\right) \geq c\left\|x y^{*}\right\|_{\infty}=c>c / 2$. Thus

$$
\frac{c}{2} \cdot \frac{Q+P}{2}+\left(\begin{array}{cc}
0 & x y^{*} \\
y x^{*} & 0
\end{array}\right) \geq 0 .
$$

However,

$$
\begin{aligned}
\frac{c}{2} \cdot \frac{Q+P}{2}+\left(\begin{array}{cc}
0 & x y^{*} \\
y x^{*} & 0
\end{array}\right) & \geq \frac{c}{2} \cdot \frac{a I+P}{2}+\left(\begin{array}{cc}
0 & x y^{*} \\
y x^{*} & 0
\end{array}\right) \\
& \geq\left(\begin{array}{cc}
(c / 4) P_{1} & x y^{*} \\
y x^{*} & (a c / 4) I
\end{array}\right),
\end{aligned}
$$

and hence the last matrix is not positive semidefinite. Since $(c / 4) P_{1}$ is positive semidefinite, we must have $(c / 4) x^{*} P_{1} x<(4 / a c)\left(x^{*} x\right)^{2}$. Thus $\lambda_{\max }\left(P_{1}\right)<$ $16 / a c$ and, similarly, $\lambda_{\max }\left(P_{2}\right)<16 / a c$. Thus $\lambda_{\max }(P)<16 /$ ac for all $P \in$ $S_{+}$, which implies that $S_{+}$is bounded.

Theorem 4.2. Let $S$ be a closed convex subset of $H_{m+n}$ such that any $P \in S$ may be written $P_{1} \oplus P_{2}$ where $P_{1} \in H_{m}$ and $P_{2} \in H_{n}$. Assume that $S$ satisfies the two conditions in Theorem 4.1. Let $N(\cdot)$ be the norm defined in (4.1), and let $N^{*}(\cdot)$ be its dual. Then for any $A \in M_{m, n}, N^{*}(A) \leq 1$ if and only if there is a matrix $R \in S^{D}$ such that

$$
R+\left(\begin{array}{cc}
0 & A \\
A^{*} & 0
\end{array}\right) \geq 0
$$

where

$$
S^{D}=\left\{R=R_{1} \oplus R_{2}: R_{1} \in H_{m}, R_{2} \in H_{n}, \text { and } \operatorname{tr} R P \leq 2 \forall P \in S\right\} .
$$

Note that the usual definition of $S^{D}$ would have the condition $\operatorname{tr} R P \leq 1$ instead of $\operatorname{tr} R P \leq 2$.

Proof. We first note that if $S$ satisfies the two conditions in Theorem 4.1 then so does $S^{D}$. Also, it is not hard to show that $S^{D D}=S$.

Let $A \in M_{m, n}$ be given. Suppose that there is a matrix $R \in S^{D}$ such that

$$
R+\left(\begin{array}{cc}
0 & A \\
A^{*} & 0
\end{array}\right) \geq 0
$$

Then for any matrix $B \in M_{m, n}$ with $N(B) \leq 1$, there is a matrix $P \in S$ such that

$$
P+\left(\begin{array}{cc}
0 & B \\
B^{*} & 0
\end{array}\right) \geq 0
$$

Thus, by (4.4) and (4.5), we have

$$
0 \leq \operatorname{tr}\left[R+\left(\begin{array}{cc}
0 & A \\
A^{*} & 0
\end{array}\right)\right]\left[P+\left(\begin{array}{cc}
0 & B \\
B^{*} & 0
\end{array}\right)\right]=\operatorname{tr} R P+2 \operatorname{Retr} A B^{*},
$$

which implies

$$
-2 \operatorname{Retr}\left(A B^{*}\right) \leq \operatorname{tr} R P \leq 2 \text { for all } B \text { with } N(B) \leq 1 .
$$

Thus $N^{*}(A) \leq 1$. 
Let $\alpha$ be the least number such that there is a positive semidefinite matrix $R \in S^{D}$ such that

$$
Z \equiv \alpha R+\left(\begin{array}{cc}
0 & A \\
A^{*} & 0
\end{array}\right) \geq 0
$$

Let $\widehat{R} \in S^{D}$ be such a matrix. We must show that $N(A) \geq \alpha$. By the choice of $\widehat{R}$, Lemma 1.4 guarantees that there is a positive semidefinite nonzero matrix $M$ in the polar or the cone of feasible directions at $\widehat{R}$ such that

$$
\left[\alpha \widehat{R}+\left(\begin{array}{cc}
0 & A \\
A^{*} & 0
\end{array}\right)\right] M=0
$$

Let

$$
M=\left(\begin{array}{ll}
M_{11} & M_{12} \\
M_{12}^{*} & M_{22}
\end{array}\right) .
$$

Because $M$ is in the polar of the cone of feasible directions at $\widehat{R}$,

$$
\operatorname{tr}\left(\begin{array}{cc}
\widehat{R}_{1}-R_{1} & 0 \\
0 & \widehat{R}_{2}-R_{2}
\end{array}\right) M \leq 0 \text { for all } R_{1} \oplus R_{2} \in S^{D}
$$

or, equivalently,

$$
\operatorname{tr}\left(M_{11} \oplus M_{22}\right) R \leq \operatorname{tr}\left(M_{11} \oplus M_{22}\right) \widehat{R} \text { for all } R \in S^{D} .
$$

Because there is a positive definite matrix $R$ in $S^{D}$, the right-hand side of (4.7) must be positive. Thus, multiplying $M$ by a positive constant if necessary, we may assume that the right-hand side of $(4.7)$ is 2 . That is

$$
\operatorname{tr}\left(M_{11} \oplus M_{22}\right) R \leq 2 \text { for all } R \in S^{D},
$$

which says that $M_{11} \oplus M_{22} \in S^{D} D$, which in turn implies that $N\left(M_{12}\right) \leq 1$. Condition (4.6) now yields

$$
2 \operatorname{Retr}\left(A^{*} M_{12}\right)=-\alpha \operatorname{tr}(\widehat{R} M)=-2 \alpha .
$$

Since $N\left(M_{12}\right) \leq 1$, this implies $N^{*}(A) \geq \alpha$.

The trace norm is defined on $M_{m, n}$ by $\|A\|_{1}=\operatorname{tr}|A|$. It is well known that the trace norm is the dual of the spectral norm. In the following corollary we apply Theorem 4.2 to the norms $\|\cdot\|_{\infty}, \omega(\cdot),\|\cdot\|_{H, \infty}$, and $\|\cdot\|_{H, \omega}$.

Corollary 4.3. Let $A \in M_{m, n}$ and $B \in M_{n}$. Then that

(a) $\|A\|_{1} \leq 1$ if and only if there are matrices $P \in H_{m}$ and $Q \in H_{n}$ such

$$
\left(\begin{array}{cc}
P & A \\
A^{*} & Q
\end{array}\right) \geq 0, \quad \operatorname{tr} P \geq 1, \operatorname{tr} Q \geq 1 .
$$

(b) $\omega^{*}(B) \leq 1$ if and only if there is a matrix $P \in H_{n}$ such that

$$
\left(\begin{array}{cc}
P & B \\
B^{*} & P
\end{array}\right) \geq 0, \quad \text { tr } P \leq 1 .
$$

(c) $\|A\|_{H, \infty}^{*} \leq 1$ if and only if there are diagonal matrices $D_{1} \in H_{m}$ and $D_{2} \in H_{n}$ such that

$$
\left(\begin{array}{cc}
D_{1} & A \\
A^{*} & D_{2}
\end{array}\right) \geq 0, \quad \operatorname{tr} D_{1} \leq 1, \operatorname{tr} D_{2} \leq 1
$$


(d) $\|B\|_{H, \omega}^{*} \leq 1$ if and only if there is a diagonal matrix $D \in H_{n}$ and a Hermitian matrix $Z \in H_{n}$ (with main diagonal not necessarily zero) such that

$$
\left(\begin{array}{cc}
D+Z & B \\
B^{*} & D-Z
\end{array}\right) \geq 0, \quad \operatorname{tr} D \leq 1 .
$$

There are other well-known norms that are of the type described in Theorem 4.1. In particular, the unitarily invariant norms on $M_{m, n}$ are such norms. Let $\|\cdot\|$ be a unitarily invariant norm on $M_{m, n}$. Then it follows from [6, Corollary 3.2] that for any $A \in M_{m, n},\|A\| \leq 1$ if and only if there are matrices $P \in H_{m}$, $Q \in H_{n}$ such that

$$
\left(\begin{array}{cc}
P & A \\
A^{*} & Q
\end{array}\right) \geq 0, \quad\|P\| \leq 1, \quad\|Q\| \leq 1 .
$$

(How to interpret $\|X\|$ in a consistent manner when $X \notin M_{m, n}$ is explained in [5].)

\section{REFERENCES}

1. T. Ando, On the structure of operators with numerical radius one, Acta Sci. Math. (Szeged) 34 (1973), 11-15.

2. T. Ando and K. Okubo, Induced norms of the Schur multiplier operator, Linear Algebra Appl. 147 (1991), 181-199.

3. R. Fletcher, Semidefinite matrix constraints in optimization, SIAM J. Control Optim. 23 (1985), 493-512.

4. R. A. Horn and C. R. Johnson, Matrix analysis, Cambridge Univ. Press, New York, 1985.

5. R. A. Horn and R. Mathias, Cauchy-Schwarz inequalities associated with positive semidefinite matrices, Linear Algebra Appl. 142 (1990), 63-82.

6. _ An analog of the Cauchy-Schwarz inequality for Hadamard products and unitarily invariant norms, SIAM J. Matrix Anal. Appl. 11 (1990).

7. O. Mangasarian, Nonlinear programming, McGraw-Hill Ser. Systems Sci., McGraw-Hill, New York, 1969.

8. R. Mathias, The spectral norm of a nonnegative matrix, Linear Algebra Appl. 139 (1990), 269-284.

9. R. McEachin, Analysis of an inequality concerning perturbation of self-adjoint operators, Ph.D. thesis, Univ. of Illinois at Urbana-Champaign, 1990.

10. I. Olkin and J. Pratt, A multivariat Tchebycheff inequality, Ann. Math. Statist. 29 (1958), 226-234.

11. S-.C. Ong, On the Schur multiplier norm of matrices, Linear Algebra Appl. 56 (1984), 45-55.

12. M. Overton, On minimizing the maximum eigenvalue of a symmetric matrix, SIAM J. Matrix Anal. Appl. 9 (1988), 256-268.

13. V. Paulsen, S. Power, and R. Smith, Schur product and matrix completions, J. Funct. Anal. 85 (1989), 151-178.

Department of Mathematics, College of William and Mary, Williamsburg, VIRGINIA 23187

Current address: Institute for Mathematics and Applications, 514 Vincent Hall, 206 Church Street SE, Univerisy of Minnesota, Minneapolis, Minnesota 55455

E-mail address: na.mathias@na-net.ornl.gov 